C Springer-Verlag Berlin Heidelberg 2011

This document is published in: C. Stephanidis (Ed.) (2011). Universal Access in $\mathrm{HCl}$ : Design for All and elnclusion: 6th International Conference, $\mathrm{UAHCl}$ 2011, Held as Part of $\mathrm{HCl}$ International 2011, Orlando, FL, USA, July 9-14, 2011, Proceedings, Part I, (Lecture Notes in Computer Science, 6765). Springer, ISBN: 978-3-642-21671-8, pp. 249-257.

DOI: http://dx.doi.org/10.1007/978-3-642-21672-5 28 


\title{
A Study of Accessibility Requirements for Media Players on the Web
}

\author{
Lourdes Moreno, María Gonzalez, Paloma Martínez, and Ana Iglesias \\ LaBDA Group, Computer Science Department, Universidad Carlos III de Madrid, \\ Avda. Universidad 30, 28911 Leganés, Madrid, Spain \\ \{lmoreno, mgonza1, pmf, aiglesia\}@inf.uc3m.es
}

\begin{abstract}
Multimedia content covers the Web, and we should provide access to all people. For this reason, we must consider including accessibility requirements in a synchronized manner with the alternative resources such as caption and audio description among others. In addition, it is very important to take into account accessibility requirements in the player to avoid barriers and to ensure access to this multimedia content as well as their resources. This paper presents an overall study on standards and players with accessibility requirements. Moreover, solutions to improve the accessibility features in the YouTube player are presented. Based on this study, we have distinguished a set of guidelines to take into account for including accessibility requirements in players. Furthermore, we suggest an agile evaluation process which indicates the order of accessibility guidelines to check. Finally, the proposed evaluation method is put into practice with a case study: accessibility features are evaluated in three widely used players.
\end{abstract}

Keywords: Web accessibility, user agent, media players, standard, evaluation, accessibility requirement.

\section{Introduction}

Multimedia content, such as video and audio, has long filled the Web and will likely continue to do so well into the future. Multimedia content must be accessible for people with disabilities according to standards like the Web Content Accessibility Guidelines (WCAG) [1] of Web Accessibility Initiative (WAI) [2]. In order to achieve multimedia content accessibility to a greater or lesser degree, synchronized alternatives such as captions, audio descriptions and transcriptions need to be provided. Additionally, the accessibility of multimedia content on the Web requires that a particular chain of essential, interdependent, and accessible components [3] should be taken into account for user agents. Specifically, media players should allow to play deliver accessible multimedia content and therefore a positive user-video interaction would success.

While accessibility problems may sometimes occur within Web content itself, other times they can be traced to the browser, assistive technologies (software used by people with disabilities to aid in their interaction with computers) or even a user's inability to handle a particular tool. This latter point is particularly troubling, since it 
is of no practical use to include an accessible video with audio description if a blind user, in the end, cannot access this audio alternative due to accessibility barriers within the media player itself. Therefore, media players should be developed according to the User Agent Accessibility Guidelines (UAAG) [4] of WAI and Universal Design criteria. Furthermore, it is imperative that Web designers are familiar with the UAAG as well as the existing media players capable of the accessible delivery of multimedia content.

\section{Background}

The User Agent Accessibility Guidelines (UAAG) explain how to make user agents including Web browsers, media players, and assistive technologies - accessible for people with disabilities, and particularly, how to increase the accessibility of Web content. In general, media players must ensure that all audio and video controls are accessible to the keyboard alone and can be accessed by a user using a screen reader. While among the two versions of the UAAG currently available, the earlier UAAG 1.0 (approved in 2002) is considered the version of reference, UAAG 2.0 is currently being developed to help make a new generation of user agents functionally accessible, to provide a gate to alternative information based on user technologies and to align itself with WCAG 2.0 (W3C Recommendation).

Following the Guideline 1.2 of WCAG 2.0 ("provide alternatives for time-based media") [1], the media content must be accompanied by media alternatives as caption (or subtitles for deaf people), audio description, sign language, etc. in synchronized media. The UAAG includes guidelines to ensure that the players provide support for these media alternatives.

User agents like players may get conformance to UAAG 2.0 at one of three conformance levels. The level achieved depends on the level of the success criteria that have been satisfied. The conformance levels are: "A" (the player satisfies all of the Level "A" success criteria), "AA" or "Double-A" (it satisfies all of the Level "A" and Level "AA" success criteria) or "AAA" or "Triple-A" (it satisfies all of the success criteria).

Current media players have, to a greater or lesser extent, accessibility features conforming to the UAAG. These players are embedded in a web page or through a standalone player. Embedding the player in a web page allows the user to access the content without another application opening as many Flash players, but the standalone players usually have more control options. In general, the standalone versions of the media players are far more accessible than the embedded versions [5].

Several media formats present caption capabilities. The most famous ones are RealPlayer ${ }^{1}$, Windows Media Player ${ }^{2}$, QuickTime ${ }^{3}$ and iTunes ${ }^{4}$. iTunes and the videos that it gets synced with iPod-family devices are capable of displaying subtitles with the ability to have elegant closed-captions (captions that can be displayed or not, depending on user preference) [6].

\footnotetext{
${ }^{1}$ RealPlayer, www.real.com

${ }^{2}$ Windows Media Player, http://windows.microsoft.com

${ }^{3}$ QuickTime, www.apple.com/

${ }^{4}$ iTuunes, www.apple.com/
} 
In relation to audio description, it is recommended for video players to have an audio description track that runs the length of the video. Then, the player may allow audio description controls for the user.

Most of the videos online are delivered via Adobe Flash-based in-page video players ${ }^{5}$. Flash has an excellent compression system that can deliver high-fidelity audio and high-resolution video without taxing bandwidth. In addition, Flash is installed in most browsers [6]. Due to this fact, this latter group of Flash media players has long been used on the Web, as YouTube Video Player ${ }^{6}$. Although the YouTube Video Player provides subtitles, it presents accessibility problems as it will show in section four.

The HTML5 [7] offers web accessibility in the accessible embedded media player a huge step forward. Basically, the new standard provides the latest commands, such as <video $>$ and <audio>, which can create and label some buttons in a way that allows keyboard shortcuts to access them, and screen readers to tell the user which buttons are available. However, HTML5 elements which provide support for the some UAAG guidelines and for the inclusion of caption and audiodescription have not been included in the new standard still under development.

A literature survey has covered a few studies describing the levels of accessibility and compliance of media players with the UAAG [8]. The application of the UAAG using the checklist for WAI-UAAG documentation [9] has been observed to be quite difficult for some developers and evaluators. That is why this paper proposes an agile evaluation process for checking accessibility features in media web players.

\section{Accessibility Evaluation Method for Media Players}

A study of accessibility requirements for media players based on the relevant sections of UAAG 2.0 (Working Draft 17 June 2010) [4] has been carried out. First, the UAAG 2.0 guidelines related to media players were selected (section 3.1). Next, an agile method for evaluating the accessibility requirements of media players is proposed based on WAI documentation (section 3.2).

\subsection{Guidelines Subset of UAAG for Media Players}

In this study, the first step was to distinguish which guidelines from UAAG are specifically related to media players (others are related to other user agents as browsers, etc.).

Table 1 indicates the number of guidelines for media players taken into account grouped by conformance levels of UAAG. Table 2 detail the selected guidelines of UAAG (third column).

\subsection{Agile Evaluation Method}

In the second step, the guidelines subset was grouped according to accessibility issues to take into account in media players. Our proposal has differentiated fourteen groups

\footnotetext{
${ }^{5}$ Flash Player penetration. Flash content reaches $99 \%$ of Internet viewers, ADOBE, December 2010, http://www.adobe.com/products/player_census/flashplayer/

${ }^{6}$ Youtube's web site: http://www.youtube.com, testing October 2010
} 
Table 1. Number of guidelines by conformance levels of UAAG for media players

\begin{tabular}{|c|c|c|c|c|}
\cline { 2 - 5 } \multicolumn{1}{c|}{} & $\begin{array}{c}\text { Level } \\
\text { "A" }\end{array}$ & $\begin{array}{c}\text { Level } \\
\text { "AA" }\end{array}$ & $\begin{array}{c}\text { Level } \\
\text { "AAA" }\end{array}$ & $\begin{array}{c}\text { Undetermined } \\
\text { in Working } \\
\text { Draft }\end{array}$ \\
\hline $\begin{array}{c}\text { Selection guidelines for media } \\
\text { players }\end{array}$ & 76 & 29 & 14 & 6 \\
\hline
\end{tabular}

Table 2. Agile evaluation method for checking accessibility in media players

\begin{tabular}{|l|l|l|}
\hline Group & \multicolumn{1}{|c|}{ Accessibility issue } & \multicolumn{1}{c|}{ Guidelines (G)and Success Criteria (sc) } \\
\hline G1 & Alternative content & G: 3.1 \\
\hline G2 & Highlighting & sc: 3.5 .1 \\
\hline G3 & $\begin{array}{l}\text { Text configuration and } \\
\text { alternative views }\end{array}$ & sc: 3.6 .1 \\
& Volume configuration & G: 3.7 \\
\hline G4 & Synthesized speech & G: 3.8 \\
\hline G5 & Viewfiguration & sc: 3.10 .4 \\
\hline G6 & Focus & sc: $3.11 .3,3.11 .4,3.11 .6,3.11 .7,3.11 .8$, \\
\hline G7 & & $3.11 .10,3.11 .11$ \\
& sc: 5.4 .2 \\
\hline G8 & Keyboard access and & sc: $3.11 .9,4.1 .1,4.1 .3,4.1 .4,4.1 .6,4.1 .7$, \\
& navigation & $4.1 .8,4.1 .9,4.1 .10,4.1 .11,4.1 .12,4.7 .5$, \\
& \multicolumn{1}{|c|}{$4.7 .6,4.7 .7$} \\
\hline G9 & Preferences & G: 4.5 \\
\hline G10 & Text search & G: 4.6 \\
\hline G11 & Toolbar configuration & G: 4.8 \\
\hline G12 & Control of content that & $4.9 .2,4.9 .5,4.9 .6,4.9 .6,4.9 .7,4.9 .8,4.9 .9$, \\
& may reduce accessibility & $4.9 .10,4.9 .11$ \\
\hline G13 & Unnecessary message & G: 5.1 \\
\hline G14 & Documentation & G: 5.3 \\
& accessibility features & \\
\hline
\end{tabular}

as it is shown in Table 2. We propose to follow the order fixed in Table 2 for an agile evaluation process.

\section{Accessibility Evaluation of Accessible Media Players}

This section presents an accessibility evaluation of three media players. The evaluation method proposed in previous section has been used for the evaluation study. The studied players are Youtube, BBC iPlayer ${ }^{7}$ and CCPlayer ${ }^{8}$, all of them

7 BBC iPlayer's web site: http://iplayerhelp.external.bbc.co.uk/help/information/about_bbc _iplayer ,testing October 2010

8 CCPlayer's web site: http://ncam.wgbh.org/invent_build/web_multimedia/tools-guidelines/ ccplayer, ccPlayer version 2.0.2, testing October 2010 
developed with Flash technology. They are embedded in a web page and all of them allow the user to access the content without another application opening.

The evaluation process has been carried out by a human evaluator who has high level of accessibility multimedia experience; the use context was with Windows 7 operating system, Mozilla Firefox Browser version 3.6.13 and the screen reader NVDA 9 .

- Group G1 (first column of Table 2): The players' behaviour in the first group of guidelines is similar. The three video players only satisfy success criteria 3.1.1 with conformance level "A" (identify presence of alternative content). Figure 1 shows screenshots of three players where the "CC" button (in red) identifies the presence of captions. The rest of success criteria of this group are not satisfied.

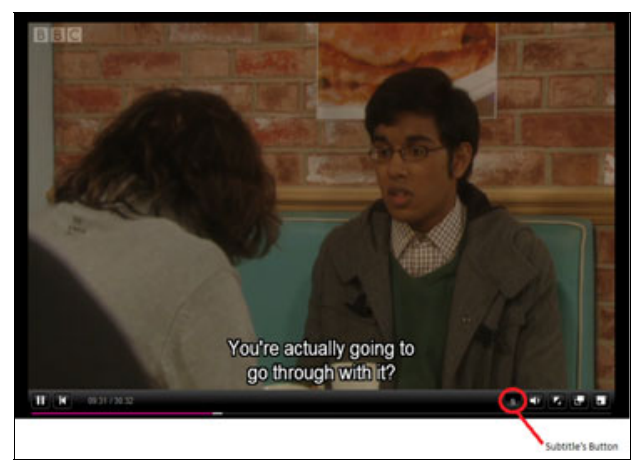

Fig. 1. BBC iPlayer's screenshot with alternative (caption) and mechanist to identify it

- Group G2: The second group is completely satisfied by the players, providing highlighting items for selection, content focus, enabled elements and visited links.

- Group G3: In the third group YouTube player is the only one that satisfies the success criteria 3.6.1 with conformance level "A", allowing the configuration of the captions through a text setting menu (see Figure 2). However, it does not allow the user to change the colour of the text, so the configuration is not completely accessible. The rest of success criteria of this group are not satisfied.

- Group G4: All players satisfy the success criteria 3.7.1 (global volume) and 3.7.2 (related to speech volume), both with conformance level "A".

- Group G5: None of the success criteria of this group (related to speech features) are satisfied.

- Group G6: The success criteria 3.10.4 (resizable characteristics, level "A") is satisfied by all the video players.

- Group G7: Every success criteria of this group is satisfied by the players, all of them with conformance level "A".

\footnotetext{
${ }^{9}$ NVDA, http://www.nvda-project.org/, retrieved October 2010
} 


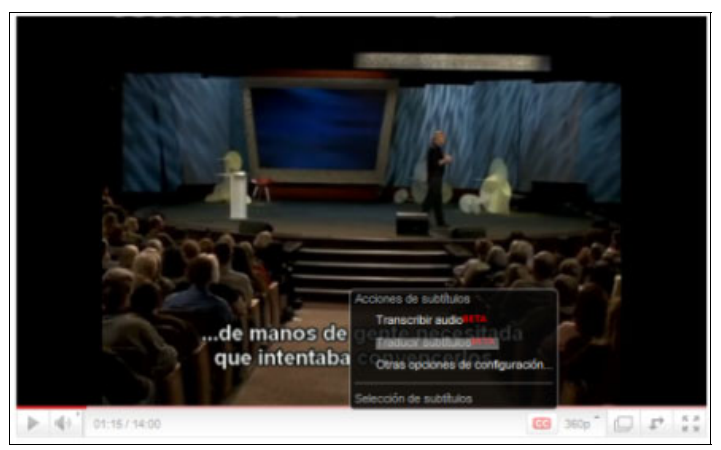

Fig. 2. YouTube's screenshot with subtitles and text setting menu

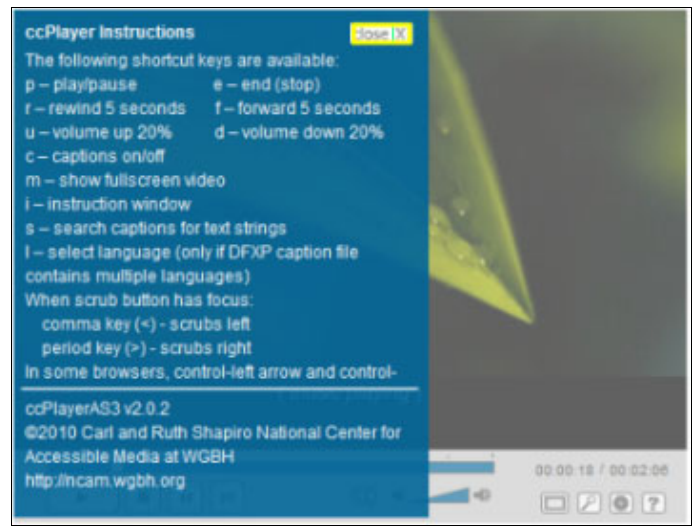

Fig. 3. CCPlayer's screenshot showing a command's menu

- Group G8: The success criteria related to keyboard and sequential navigation are satisfied by all players $(3.11 .9,4.1 .1,4.1 .3,4.1 .4$ of level " $\mathrm{A}$ " and 4.1.8, 4.7.5 of level "AA"). However, the guidelines related with commands (4.1.6 of level " $A$ " and 4.1.7, 4.1.9 of level "AA") are only satisfied by CCPlayer, which shows an explicative menu with all the shortcut keys as it is shown Figure 3. The rest of success criteria are not satisfied.

- Group 9: Only the success criteria 4.5.1 (change preference settings, level " $\mathrm{A}$ ") is satisfied by all the video players. The success criteria 4.5.2 (persistent accessibility settings, level "A") is satisfied by YouTube player and BBC iPlayer, although Youtube only keeps the size of the player and it does not keep the subtitles. The rest of success criteria are not satisfied.

- Group 10: CCPlayer is the only player that satisfies all success criteria of the tenth group. It has a caption search (see Figure 4).

- Group G11: The guideline 4.8 is not satisfied by the players. This group references the position configuration (4.8.1) and restoration of default toolbars (4.8.2), both with level AAA. 


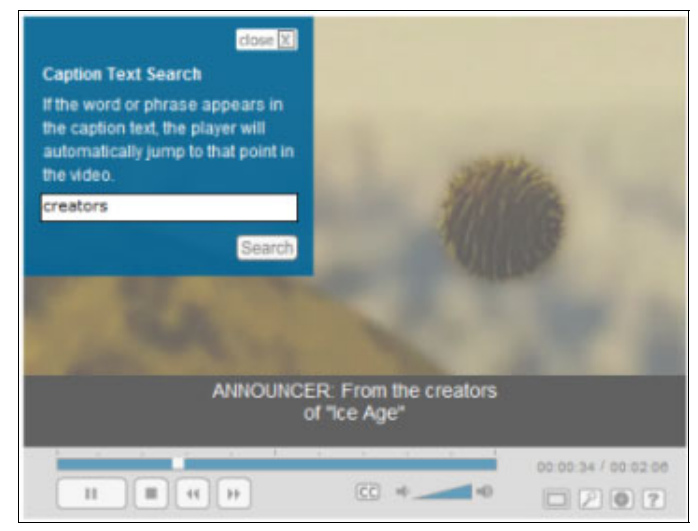

Fig. 4. CCPlayer's screenshot with caption search

- Group G12: Some of the success criteria are satisfied such as time-based media load-only (4.9.2), stop/pause/resume multimedia (4.9.6), navigate multimedia $\left(4.9 .6^{10}\right)$, all of them level "A", semantic navigation of time-based media (4.9.7) and sizing playback viewport (4.9.9) with level "AA". The rest of success criteria are not satisfied.

- Group G13: All players satisfy the success criteria 5.1.2 (retrieval progress, level "A"). The success criteria 5.1.1 (option to ignore, level "AA") is satisfied by YouTube player and CCPlayer. When a text message appears during the reproduction, you can turn it off with these video players.

- Group G14: The success criteria 5.3.6 (appropriate language, the undetermined conformance level) is not satisfied by the video players. The rest of success criteria are only satisfied by CCPlayer, NCAM's site provides a large amount of documents based on CCPlayer.

\subsection{Discussion}

As we can observe in the percentages obtained in Table 3, CCPlayer offers the best results in each one of the priority levels.

Therefore, we can conclude that CCPlayer is the most accessible player. It does not only offer accessibility but it also offers the user different documents among which are described accessibility features to use on it. Moreover, it is the only one that has a menu which explains the keyboard shortcuts and it allows searching for any word in the video. Furthermore, this player is compatible with screen readers but audio description examples are not found.

YouTube player provides subtitles. However, it has difficulty operating player controls in some browsers and they are not accessible by the keyboard. Another problem is that screen reader tools for the visually impaired people cannot always distinguish accurately the function of controls implemented in Flash, and some screen

${ }^{10}$ In UAAG 2.0 (W3C Working Draft 17 June 2010) the guideline 4.9.6 is repeated with two different success criteria. 
readers cannot access controls at all. With the objective of achieving an accessible YouTube Video Player, complementary technological solutions have been developed that we provide following.

The way to get around keyboard and screen reader access is through JavaScript API, which talks to the video player, while providing regular HTML controls for keyboard and screen reader users. YouTube has JavaScript API [10], but we have observed that this API does not provide caption support. Other JavaScript API has been found [11].

Others accessible solutions for YouTube Video Player are "Accessible Easy YouTube Player" [12] and "Accessible YouTube player controls" [13]. The first solution allows users to paste the URL and then use an accessible player to show the video, this player provides an accessible interface with accessible controls as shown in Figure 5, however it does not provide navigation buttons or controls to move backward and forward just as ATAG say. In this case of "Accessible YouTube player controls", the controls have been re-coded given the fact that they are more accessible to keyboard and screen reader users than the standard YouTube controls. Finally, more resources have been found to adding closed captions to YouTube videos [14][15].

YouTube player is working on a beta version with subtitle's translation, audio's transcription and setting options, as the subtitle's font. There are also some videos audio-description is embedded, but the audio-description control is missed.

BBC iPlayer allows providing subtitles. BBC includes in TV programming approximately twenty- five hours with audio description per week in different genres. However, similar to the other players, the user cannot control to play or not the audio description signal.

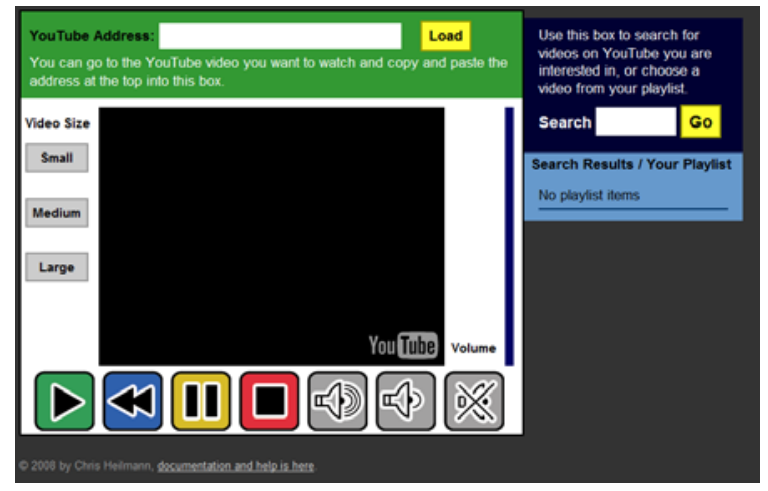

Fig. 5. Accessible Easy YouTube Player Interface [12]

Table 3. Percentage of the guidelines satisfied by players, grouped by conformance level

\begin{tabular}{|c|c|c|c|}
\cline { 2 - 4 } \multicolumn{1}{c|}{} & Level "A" & Level "AA" & Level "AAA" \\
\hline YouTube & $66.67 \%$ & $25 \%$ & $0 \%$ \\
\hline BBC iPlayer & $63.89 \%$ & $20 \%$ & $0 \%$ \\
\hline CCPlayer & $75 \%$ & $45 \%$ & $11.11 \%$ \\
\hline
\end{tabular}




\section{Conclusions}

The amount of multimedia content on the Web continues increasing, so the accessibility of media players and their access through the Web site become more and more important. This paper presents a study directed to web professionals of accessible media players, including both in depth documentation and the results of experimentation conducted on diverse video players.

The study demonstrates that some existing media players include accessibility features but currently none of them is enough accessible.

Acknowledgments. This research work is supported by the Research Network MAVIR (S2009/TIC-1542 (see www.mavir.net/)), and GEMMA (TSI-020302-2010141) and SAGAS (TSI-020100-2010-184) research projects.

\section{References}

1. W3C, WAI, Web Content Accessibility Guidelines (WCAG) (2010), http: / / www .w3 . org/WAI/intro/wcag.php

2. W3C, Web Accessibility Initiative (WAI) (2010), http: / /www . w3 . org /WAI /

3. Moreno, L., Martínez, P., Ruiz-Mezcua, B.: Disability Standards for Multimedia on the Web. IEEE Multimedia 15(4) (2008) ISSN: 1070-986X

4. W3C, WAI, User Agent Accessibility Guidelines (UAAG) (2010), http: / / www.w3 . org/WAI/intro/uaag . php

5. WebAIM, Media Player Accessibility (2010), http: / /webaim.org/techniques / captions /mediaplayers /

6. CANnect, Accessible Video and Audio, How-To Guide for Creating Accessible Online Learning Content (2010),

http://sloanconsortium.org/cannect/projectone/advice/videoaudio.php

7. W3C, HTML5, A vocabulary and associated APIs for HTML and XHTML http: / /Www.w3 .org/TR/2009/WD-htm15-20090423/

8. Watanabe, T., Umegaki, M.: Capability survey of user agents with the UAAG 1.0 test suite and its impact on Web accessibility. Universal Access Inf. Soc. 6(3), 221-232 (2007)

9. W3C, WAI, UAAG 2.0, Checklist (for Authoring Tool Accessibility Guidelines 2.0), http: / /www.w3 .org/TR/2009/WD-ATAG20-20090521/checklist.html

10. YouTube JavaScript Player API Reference, http://code.google.com/apis/YouTube/js_api_reference.html

11. OSU Web Accessibility Center's, Captioning YouTube and Providing Accessible Controls, http: / /wac . osu.edu/examples/YouTube-player-controls /

12. Christian Heilmann's Accessible Easy YouTube http://icant.co.uk/easyYouTube /

13. Accessible YouTube player controls (vision australia)

http: / /www.visionaustralia.org/info.aspx?page $=2260$

14. YouTubeCC, http://www. YouTubecc.com/

15. CaptionTube http: / / captiontube.appspot.com/ 